\title{
A cloud-based pervasive application for monitoring oxygen saturation and heart rate using fuzzy-as-a-service
}

\author{
Bhavesh Pandya \\ School of Science and Technology \\ Nottingham Trent University \\ Nottingham, United Kingdom \\ bhavesh.pandya@ntu.ac.uk
}

\author{
Amir Pourabdollah \\ School of Science and Technology \\ Nottingham Trent University \\ Nottingham, United Kingdom \\ amir.pourabdollah@ntu.ac.uk
}

\author{
Ahmad Lotfi \\ School of Science and Technology \\ Nottingham Trent University \\ Nottingham, United Kingdom \\ ahmad.lotfi@ntu.ac.uk
}

\begin{abstract}
Due to the rapid development of information technology, health monitoring research demands are growing tremendously. People prefer to use wearable devices that consist of sensors, which help in monitoring the health of individuals. This study aims to develop a system that remotely monitors the physiological aspects of individuals, such as blood oxygen saturation and pulse measurement, using wearable sensors and a new cloud-based fuzzy logic system, and generates necessary alarms. Monitoring health remotely based on wearable and non-invasive sensors provides a cost-effective solution, which in turn permits the elderly people to continue to live in his/her home environment as opposed to spending on expensive healthcare facilities. The developed system shall permit smooth physiological monitoring of an individual's health by healthcare personnel in real-time. The developed system will not only assess health conditions but also provide feedback from distant facilities. Moreover, the developed rule-based fuzzy system can infer an individual's health condition. The study uses pulse rate and oxygen saturation data, which are processed by a fuzzy system on the cloud, and the acquired results are displayed on a mobile application.
\end{abstract}

\section{CCS CONCEPTS}

- Computing methodologies $\rightarrow$ Vagueness and fuzzy logic; • Computer systems organization $\rightarrow$ Cloud computing.

\section{KEYWORDS}

Pulse oximeter, oxygen saturation, $\mathrm{SpO}_{2}$, fuzzy logic, activity monitoring.

\section{ACM Reference Format:}

Bhavesh Pandya, Amir Pourabdollah, and Ahmad Lotfi. 2021. A cloud-based pervasive application for monitoring oxygen saturation and heart rate using fuzzy-as-a-service. In The 14th PErvasive Technologies Related to Assistive Environments Conference (PETRA 2021), fune 29-fuly 2, 2021, Corfu, Greece. ACM, New York, NY, USA, 7 pages. https://doi.org/10.1145/3453892.3453998

Permission to make digital or hard copies of all or part of this work for personal or classroom use is granted without fee provided that copies are not made or distributed for profit or commercial advantage and that copies bear this notice and the full citation on the first page. Copyrights for components of this work owned by others than the author(s) must be honored. Abstracting with credit is permitted. To copy otherwise, or republish, to post on servers or to redistribute to lists, requires prior specific permission and/or a fee. Request permissions from permissions@acm.org.

PETRA 2021, June 29-fuly 2, 2021, Corfu, Greece

(C) 2021 Copyright held by the owner/author(s). Publication rights licensed to ACM ACM ISBN 978-1-4503-8792-7/21/06 ..\$15.00

https://doi.org/10.1145/3453892.3453998

\section{INTRODUCTION}

Owing to the progression in the discipline of medicine, the older adults population rises all over the world together with developed countries thanks to immunisation, superior healthcare conveniences and treatments[12]. This, in turn, results in an increased demand for healthcare facilities in addition to medical staff. Enormous evolution in the electronics industry, for the support of human life, led to the origin of various healthcare devices and sensors [11], namely peacemakers, sphygmomanometers, and electronic stethoscopes. One of the rapidly developing fields is the application of light-weight wearable technologies to monitor human's health conditions on a daily basis [12]. In contrast, for example, electrocardiograms that are present in hospitals need electrodes, which need to be affixed to a human body, and human interference to ensure the functioning of machines. A pulse oximeter is another example of non-invasive devices that are used to uninterruptedly scrutinize oxygen saturation $\left(\mathrm{SpO}_{2}\right)$, and heart rate [10].

All over the world, life expectancy has shown tremendous improvements in healthcare caused by rising perception concerning individual and ecological hygiene [19]. According to the World Health Organisation (WHO), the older adults population $\geq 65$ years are expected to be more than children $<5$ years [13]. Note that this massive ageing population would lead to a noteworthy influence on society's social as well as economic structure in terms of healthcare needs. Moreover, healthcare service-associated cost rises because of the rising cost of pharmaceutical drugs and devices [17]. Thus, it is necessary to acquire and realize new tactics and know-how for offering superior quality healthcare services at a cheaper rate to the ageing population, thereby ensuring maximum comfort. Previously, a common method that was used for measuring plasma $\mathrm{O}_{2}$ diffusion was arterial plasma gas measurement. Arterial plasma gas is a type of plasma test comprising deflating an artery by means of a tinny syringe and removing certain blood from the body. Such a method is insensitive, affluent, not simple and excruciating.

Over time, it has been observed that living standards of people have improved tremendously, specifically when it comes to health-related qualities. As plasma oxygen overload is an essential physiological feature, acquiring real-time monitoring is absolutely essential. Recently, by means of IoT and signal processing techniques, wearable non-invasive plasma $\mathrm{O}_{2}$ scrutinising has turned out to be possible $[6,8,22]$. People can compute their plasma $\mathrm{O}_{2}$ saturation, pulse rate, etc. at their residence and acquire data about the changes that take place in their breathing and arterial oxygen saturation. Wearable plasma $\mathrm{O}_{2}$ diffusion and systems that measure pulse rates have been familiarised via radio-communication networks to team up with distributed IoT resources [7]. 
To process the collected data from sensors, many Machine Learning tools and techniques are employed. In the research reported here, fuzzy logic systems are employed. They are designed to map an input space into an output space, when the mapping simulates human-like decisions. Fuzzy logic handles uncertain concepts in the form of linguistic variables such as "little", "decent" and "very", and associated fuzzy sets to each such variables with membership grades between 0 and 1 . When compared with human cognitive traits, fuzzy logic has several advantages, specifically in situations that involve decision support making such as those needed in medical applications [16].

The main contributions of this research are as follows:

- A cloud-based fuzzy system for non-invasive real-time human health monitoring and anomaly detection. To the best of our knowledge, fuzzy systems in the form of cloud-based web services (called Fuzzy-as-a-Service here) has been utilised for the first time for healthcare purposes in our methodology based on IEEE 1855-2016 standard for fuzzy system data exchange;

- The openness of the proposed architecture allows the system's underlying knowledgebase to be easily updatable based on the health experts' recommendations without any hardware/software change on the users side;

- The openness and cloud-based nature of the proposed system can also help efficiently handling a big amount of data for multi-user scenarios. Hence, in this paper, the proposed system is evaluated using data acquired from wearable lightweight oximeter. However, the proposed system can still operate for different sensing sources;

The remainder of this paper is structured as follows: Section 2 focuses on an extensive related work that has been performed in fuzzy logic web services for real-time monitoring of $\mathrm{SpO}_{2}$ and pulse rate via sensors. Section 3 elaborates on various methodologies that are used in this study, such as data collection, data processing, XML parser, etc., along with the block diagram of the proposed system. Section 4 elucidates on experimental results that are performed in this study. Section 5 concludes the study with scope for future work.

\section{RELATED WORK}

Tremendous research has been conducted in fuzzy logic web services for real-time monitoring of pulse rate and $S p \mathrm{O}_{2}$ by means of a sensor-based oximeter. Some of the significant contributions concerning this field has been discussed below.

Authors in [4], implemented a low-cost, non-invasive Arduino UNO-based wearable health monitoring system to continuously measure the blood oxygen level $\left(\mathrm{SpO}_{2}\right)$, body temperature and heart rate. A pulse oximetry sensor was used for estimating plasma $\mathrm{O}_{2}$ level percentage. Also, LEDs and sample and hold circuits were used, thereby giving elevated accurate results on all skin tones and taking into account different finger width wherein the measurement was carried. The measured result was displayed on an LCD and transmitted on an Android mobile via HC-05 Bluetooth module. ESP8266 Wi-Fi shield was used in order to link the sensor system to the Internet and revise vital reading on the server for a particular individual. Compared to commercially available pulse oximeter
ChoiceMMed, a 2\% deviation was observed in the proposed system, thus proving the high accuracy of the proposed system.

The work reported in [20] proposed an affordable, convenient wrist-based pulse oximeter for tracking cardiac activities like $\mathrm{SpO}_{2}$ and heart rate. The pulse oximeter was embedded into a wristwatch, which in turn was serially connected to ATmega328p microcontroller for real-time processing. MAXREFDES117 pulse oximeter sensor was used, which provided easy integration with microcontroller and had a built-in level translator and power converter, thereby making the design simple. Also, using HC-05 Bluetooth module, the measured data could be displayed on the nearest smartphone or transferred to a laptop/desktop wherein necessary preprocessing could be performed on MATLAB to obtain a clean PPG signal. Tests were carried out on 200 volunteers where the device gave satisfactory results, thus validating the device.

A non-invasive Arduino-based hypoxic symptoms detection device using fuzzy has been proposed in [10]. The use of the noninvasive technique hampered the result of initial symptoms of hypoxia; therefore, Sugeno Fuzzy method tool was used to detect mild, moderate and severe hypoxia. The device consists of Max30100 sensor integrated with Arduino and a Bluetooth module for transferring data to the smartphone. Twelve trails were carried out, resulting in $2.96 \%$ error for $\mathrm{O}_{2}$ saturation and $2.86 \%$ error for heart rate. In addition, $100 \%$ accuracy was obtained from the fuzzy method. Furthermore, usage of other method and addition of variation in the input to the fuzzy method would result in more meticulous and accurate results.

The work reported in [23] presented an IoT-based wearable pulse oximeter to measure physiological data such as plasma $\mathrm{O}_{2}$ saturation $\left(\mathrm{SpO}_{2}\right)$, perfusion index (PI) and pulse rate (PR) via signal processing to evaluate data. The accuracy of the entire system was validated by conducting experiments on a simulator and an application to display the result on mobile devices. The output of the device was compared with two commercially available pulse oximeter ChoiceMMed and Innova. For $S p \mathrm{O}_{2}$ above $90 \%$, the proposed design provided similar results compared to those of ChoiceMMed and Innova with error within the range of 1 . For lower values of $\mathrm{SpO}_{2}$, the higher error rates max deviation of 3 were observed by the other two devices.

An Android-based application on health monitoring using fuzzy logic [19]. The user needed to enter their name and age in the biodata interface of the application and select data to obtain blood saturation level, pulse rate and body temperature via Bluetooth from a nearby wearable device. Fuzzy rules were designed taking into consideration the age, blood saturation level, pulse rate and body temperature, thus displaying the output as unwell, not healthy and healthy based on the fuzzy rules for each parameter. Experimental results showed $79.688 \%, 71.875 \%$ and $50 \%$ accuracies for pulse rate, oxygen saturation and body temperature, respectively.

Authors in [3] developed an IoT-based compact, low power, inexpensive and wearable $\mathrm{SpO}_{2}$ device for health monitoring. A finger sensor and an oximeter were used to measure the oxygen saturation $\left(\mathrm{SpO}_{2}\right)$ and heart rate, and the measured data was stored in a database on the server via node MCU. The measured data was available on the website (real-time) wherein a doctor/hospital could monitor a patience's status. For performance analysis of the device, the output was compared to a Mindray patient monitor (standard 
clinical instrument), wherein the difference was $2.8 \mathrm{bpm}$ for heart rate and $\pm 1.5 \%$ for $\mathrm{SpO}_{2}$. Transmission delay was calculated by transmitting data 15 times over Wi-Fi with $10 \mathrm{Mbps}$ bandwidth and resulted in an average delay of 3 seconds.

A system to measure oxygen saturation using a pulse oximeter using fuzzy logic is proposed in [5]. In this study, the calibration device was not used, thus making the system inexpensive, but using fuzzy logic, the saturation curve was plotted. For fuzzy model absorption, coefficients of a healthy person and $\mathrm{R}$ value obtained from the photodiode were used. Also, a linear relationship was formed between $\mathrm{R}$ and $\mathrm{SpO}_{2}$ with absorption coefficients using linear regression, and the $\mathrm{SpO}_{2}$ value obtained was compared with that of fuzzy logic. The result showed that the output of fuzzy logic was far more accurate and reliable as compared to the linear regression model.

An IoT-based system for measuring athletes' plasma $\mathrm{O}_{2}$ saturation and pulse rate during sports training has been developed and reported in [7]. Near IR portable tissue oximeter with STM32 microprocessor for detection was used to measure the two parameters. These parameters were then transmitted to the server via mesh nodes, which used the IEEE 802.15.4 protocol. Also, these two parameters were sent to a doctor or coaches via GPRS/Zigbee/Wi-Fi processed by the detection module, thus indicating the condition of the athlete. Additionally, the data could be ported on smartphones via GPRS/Wi-Fi. The proposed design result was compared and existing device (Philip watch) with standard medical measuring device as a reference. The result obtained was highly accurate and stable over existing devices.

The impact of plasma $\mathrm{O}_{2}$ saturation and a fuzzy logic system on inspired $\mathrm{O}_{2}$ prediction for a ventilator system has been analysed in [18]. Statistical pairwise analyses were performed to determine the correlation in a physiological parameter from scientific and medical data for updating fuzzy variables using Mamdani model to predict $\mathrm{FiO} 2$, which is fed to the ventilator to preserve $S p \mathrm{O}_{2}$ within a required level. $\mathrm{R}$ programming was used to model the fuzzy system with $75 \%$ of clinical data utilized for training and remaining $25 \%$ was utilized for testing. The output of the predictive system showed $<5 \%$ error compared to an acceptable value.

By considering the above-mentioned detailed and illustrative explanations about real-time human health monitoring in addition to its limitations, a fuzzy logic-based web service is proposed that helps in detecting a real-time human health situation via a wearable $\mathrm{SpO}_{2}$ and heart rate sensors. Unlike current systems, the proposed system makes limited use of both hardware and software. Hence, the proposed system in this paper does not require high computational resources compared to other previous works. This increases the usability and deployability features of the proposed approach in real-life scenarios.

\section{METHODOLOGIES}

The system flow illustrated in Figure.1, the output acquired from an oximeter sensor will be directly passed to a data processing block. Fuzzy variables and membership functions defined and processed by a rule-based fuzzy logic system and its output are displayed on an Android application. The proposed system which is described in

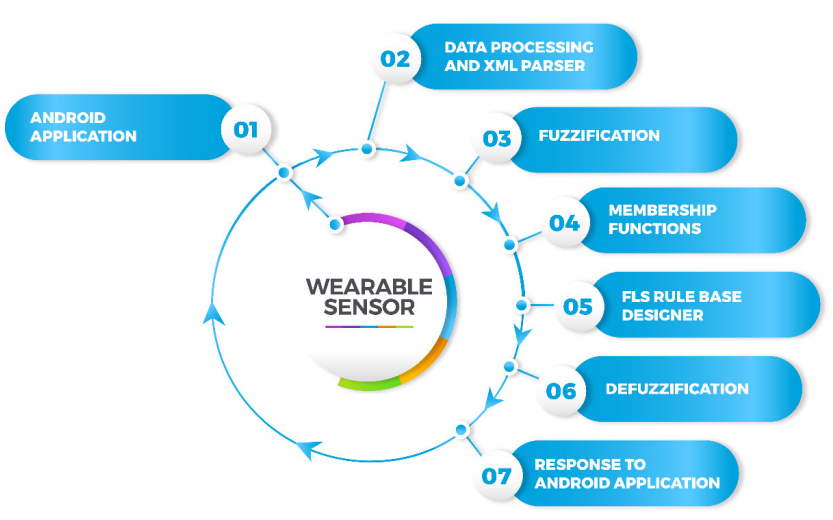

Figure 1: Flowchart of the proposed system.

Figure. 2 is developed in such a way that it will monitor an individual's health conditions by means of values that are acquired from heart rate and $\mathrm{SpO}_{2}$, which acts as the measurement parameters for managing health conditions.

\subsection{Data Collection from wearable Sensor}

For data collection, BM2000A SmartCare Oximeter wearable wrist pulse oximeter sensor was used ${ }^{1}$. The sensors, namely $S p O_{2}$ and heart rate, were used which has shown in Table1. Sensor details with the respective error code are as follows:

- $\mathrm{SpO}_{2}$ status is 0 if everything is ok.

- 0x01: $\mathrm{SpO}_{2}$ sensor not connected

- 0x02: No finger detected in sensor

- 0x04: Could not detect pulse

- 0x08: Indicates the pulse beats

Pleth is the plethysmograph signal shown on the screen of the oximeter and on the app. The RED/IR ADC values are the raw values from the analogue-to-digital converters for the red and infrared sensors in the probe. These are indeed used to calculate the $\mathrm{SpO}_{2}$ value. Perfusion index is the ratio of the pulsatile blood flow to non-pulsatile.

The sensor receives the input from a human finger that provides information about pulse rate and $\mathrm{SpO}_{2}$, which shown in Table 1. Once the input is received from the sensor, it is processed by the fuzzy system to determine the status of human health. Once the pulse rate and $\mathrm{SpO}_{2}$ data are given to the fuzzy logic system, it will process the data in a sequential manner. The rules governing the fuzzy logic processing, that can classify a situation as critical, alert or normal, are shown in Table 2.

${ }_{1}^{1}$ More detail about the sensor is available from:
http://http://devices.smartcareanalytics.co.uk/ 


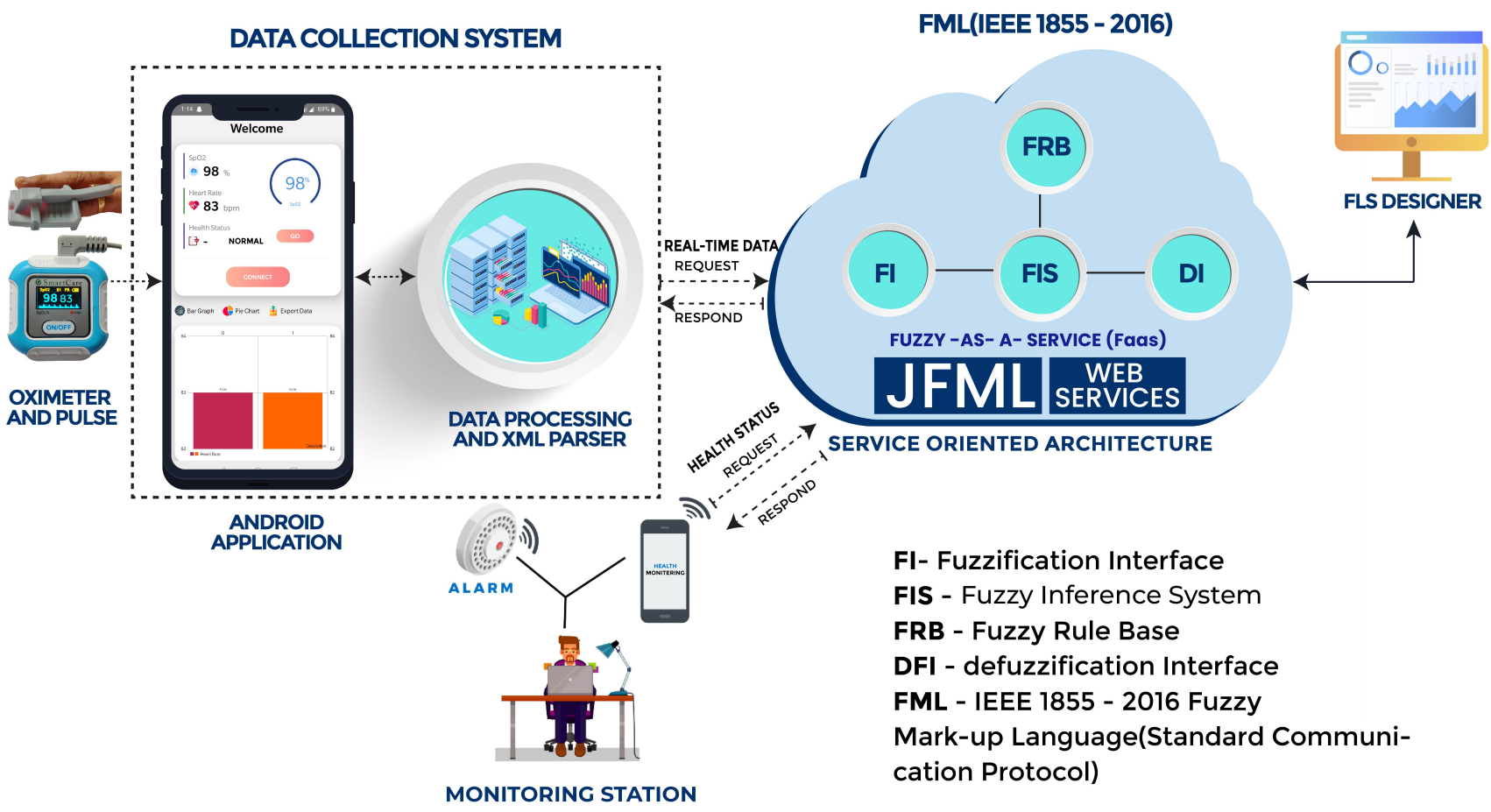

Figure 2: Framework of the proposed architecture including data collection point, data processing, monitoring point, design point and fuzzy logic system

\subsection{Data processing}

After receiving data from an Android-based wearable device, $\mathrm{SpO}_{2}$ and heart rate data can be transfer to the cloud server, where Fuzzy Logic System (FLS) file is created. It is required to set the sensor data as input values in the fuzzy system for different variables so that the system can give us the desired output. Two main API function call are designed for data exchange as follows:

setInput(): The API function call "setInput" is used for sending one or more input values to the cloud server. These values are saved at the cloud server so that the fuzzy system can evaluate the end result using the rules stored in its knowledgebase.

getOutput():This API function call is used by the user to receive output values of the FLS by request. The requested output is sent as a fuzzy set or defuzzified values. In getOutput service we intend to find out the result/output of all the data provided in the createFLS and setInput operation. The Fuzzy system evaluates all the input values for the unique URI and based on the rulebase decides the final outcome.

\subsection{Fuzzy system implementation}

The system architecture is designed to track health conditions using values extracted from heart rate and oxygen saturation which can be used as a health condition measurement system. The wearable device's output value in the application should be sent and processed using fuzzy logic.

Fuzzification turns input signals into their membership grades to the pre-defined fuzzy sets (critical, alert, low and normal). The system is a dual input system which contains $\mathrm{SpO}_{2}$ and heart rate, so that the fuzzification is repeated for both inputs. Memberships in a scheme are drawn and every membership is classified as a three turning points with different oximeter values to monitor health condition usually critical in $<30$, alert in between $>30$ and $<60$ and normal in $>60$.

- Fuzzy input set: $\mathrm{SpO}_{2}$ is the first input which is fuzzified using four fuzzy sets critical, alert, low and normal. The overall range for $\mathrm{SpO}_{2}$ is from 0 to 100 . Pulse rate is the second input which contains 4 values such as critical, normal, low and alert. The range for pulse rate input is 0 to 180 .

- Compounding: As shown in Figure.3(a), there are four fuzzy sets associated to oxygen saturation membership, critical which is less than $85 \%$, alert which is between $86 \%$ to $90 \%$, low is $91 \%$ to $94 \%$ and normal is above $95 \%$.

A membership function of the pulse rate sensor is shown in Figure. $3(\mathrm{~b})$. There are four fuzzy sets for pulse rate: critical is from 0 to $60 \mathrm{bpm}$, normal is between $60 \mathrm{bpm}$ to $90 \mathrm{bpm}$, low is between $90 \mathrm{bpm}$ to $100 \mathrm{bpm}$ and alert is above $100 \mathrm{bpm}$. 
Table 1: Dataset with a few instances of feature-extracted data.

\begin{tabular}{|c|c|c|c|c|c|c|c|}
\hline $\begin{array}{l}\text { TIMESTAMP } \\
\text { (MilliSeconds(MS)) }\end{array}$ & $\begin{array}{l}\text { PULSE } \\
\text { BPM }\end{array}$ & $\begin{array}{l}\mathrm{SpO}_{2} \\
\text { PCT }\end{array}$ & $\begin{array}{l}\mathrm{SpO}_{2} \\
\text { STATUS }\end{array}$ & PLETH & RED_ADC & IR_ADC & $\begin{array}{l}\text { PERFUSION } \\
\text { INDEX }\end{array}$ \\
\hline 0 & 87 & 97.7 & 0 & 25684 & 193775 & 250301 & 7.8 \\
\hline 10 & 87 & 97.7 & 0 & 27597 & 193872 & 249747 & 7.8 \\
\hline 20 & 87 & 97.7 & 0 & 29524 & 194064 & 250321 & 7.8 \\
\hline 30 & 87 & 97.7 & 0 & 31383 & 194311 & 249979 & 7.8 \\
\hline 40 & 87 & 97.7 & 0 & 33099 & 194559 & 251033 & 7.8 \\
\hline 50 & 87 & 97.7 & 0 & 34598 & 195154 & 252450 & 7.8 \\
\hline 60 & 87 & 97.7 & 0 & 35836 & 195384 & 252363 & 7.8 \\
\hline 70 & 87 & 97.7 & 0 & 36778 & 195897 & 253078 & 7.8 \\
\hline 80 & 87 & 97.7 & 0 & 37403 & 196275 & 253831 & 7.8 \\
\hline . & . & . & . & . & . & . & 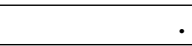 \\
\hline . & . & . & . & . & . & . & . \\
\hline . & . & . & . & . & . & . & . \\
\hline 124490 & 80 & 99.4 & 0 & 35096 & 273595 & 237903 & 1.4 \\
\hline 124500 & 80 & 99.4 & 0 & 36308 & 273428 & 237995 & 1.4 \\
\hline 124510 & 80 & 99.4 & 0 & 37626 & 273529 & 238138 & 1.4 \\
\hline 124520 & 80 & 99.4 & 0 & 39009 & 273432 & 237897 & 1.4 \\
\hline 124530 & 80 & 99.4 & 0 & 40407 & 273272 & 237526 & 1.4 \\
\hline 124540 & 80 & 99.4 & 0 & 41766 & 273303 & 237676 & 1.4 \\
\hline 124550 & 80 & 99.4 & 0 & 43067 & 273246 & 237656 & 1.4 \\
\hline 124560 & 80 & 99.4 & 0 & 44256 & 273398 & 237882 & 1.4 \\
\hline 124570 & 80 & 99.4 & 0 & 45324 & 273273 & 237634 & 1.4 \\
\hline 124580 & 80 & 99.4 & 0 & 46227 & 273077 & 237433 & 1.4 \\
\hline
\end{tabular}

Table 2: Rules for FLS

\begin{tabular}{|c|c|c|c|}
\hline No & $\mathrm{SPo}_{2}$ & Pulse & Health Status \\
\hline 1 & Critical & Critical & Critical \\
\hline 2 & Critical & Normal & Alert \\
\hline 3 & Critical & Low & Alert \\
\hline 4 & Critical & Alert & Critical \\
\hline 5 & Alert & Critical & Critical \\
\hline 6 & Alert & Normal & Normal \\
\hline 7 & Alert & Low & Normal \\
\hline 8 & Alert & Alert & Alert \\
\hline 9 & Low & Critical & Alert \\
\hline 10 & Low & Normal & Normal \\
\hline 11 & Low & Low & Normal \\
\hline 12 & Low & Alert & Normal \\
\hline 13 & Normal & Critical & Alert \\
\hline 14 & Normal & Normal & Normal \\
\hline 15 & Normal & Low & Normal \\
\hline 16 & Normal & Alert & Normal \\
\hline
\end{tabular}

A membership function of health status is shown in Figure. $3(\mathrm{c})$. There are three fuzzy sets for health status, critical is from $0 \mathrm{bpm}$ to $30 \mathrm{bpm}$, alert is from $30 \mathrm{bpm}$ to $60 \mathrm{bpm}$ and normal is from $60 \mathrm{bpm}$ to $100 \mathrm{bpm}$.

Rule Base: To perform this experiment, a total of 16 rules are created for identifying health status of human. (table:2).
Defuzzification: Defuzzification is the method that transforms a fuzzy output set into a crisp value. According to the inputs, fuzzy sets and rulebase, the output of the fuzzy system offers three classes, i.e., critical, alert and normal. Figure 3(c) shows the memberships grades for these three output fuzzy sets. The well-known centroid defuzzification method is used to convert the fuzzy output to crisp values. Once this is created, any output below 30 is considered to be critical, between 30 to 60 is considered to be alert and above 60 is treated as normal.

\subsection{Software Oriented Architecture}

It is a collection of software modules that are autonomously linked, coupled loosely and reusable. Users who have little or no dependency on software and hardware typically use SOA [14]. In our architectural solution, SOA is a web-based service that distributes the main services for fuzzy logic systems on more than one client and servers that can reach multiple users. This is why it is called Fuzzy-as-a-Service (FaaS). FaaS possesses the following built-in flexibility's:

a) Cloud service is created independent of a single fuzzy logic system.

b) Several fuzzy logic systems can be handled concurrently.

Additional details about SOA, how data transmission takes place to the system by the sensors (i.e. IoT), and how this data is stored on the cloud can be found in [9],[15],[16].

IEEE 1855-2016 is an XML-based language that facilitates the modelling of an FLS in a human-understandable, platform-independent 


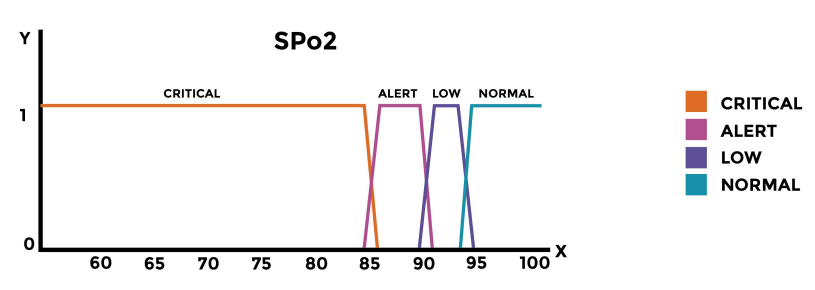

(A):MEMBERSHIP FUNCTION OF OXYGEN SATURATION (\%)

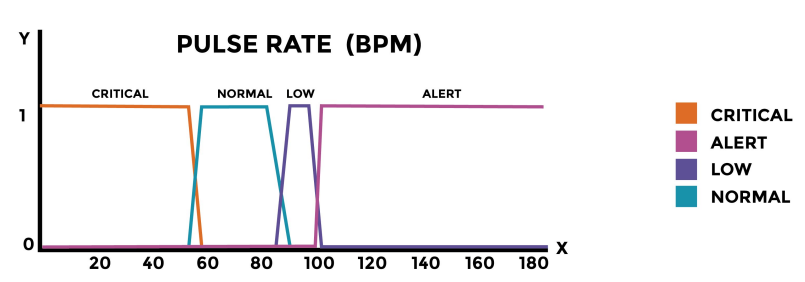

(B):MEMBERSHIP FUNCTION OF PULSE RATE (BPM).

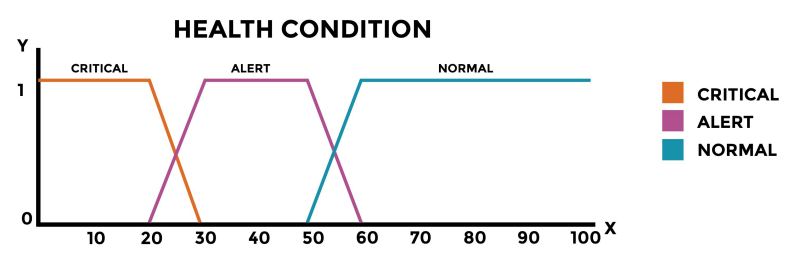

(C):DEFUZZIFICATION OF HEALTH CONDITION.

Figure 3: Membership for fuzzy inputs:(a) $S p O_{2}$ (b) Pulse rate and membership for output (c) Health Condition [19]

and web-compatible format, and it is provided in [2] and standardised in [1]. JFML is a new FML-based open-source library (IEEE 1588-2016) for fuzzy logic computations. A Web application server is built to serve the necessary HTTP messages to/from clients according to the FaaS model. JFML was used by [21] on the built-in application server for fuzzy logic computation. The framework utilises Apache Tomcat as the default Java server application. This configuration is entirely on the AWS server-side, and can be used for various purposes, especially in smart environments where client agents are sensors and/or actuators and monitoring stations.

\section{EXPERIMENTAL RESULTS}

In this study, the wearable sensor BM2000A wrist pulse oximeter was used to collect real-time data. The data is collected from four participants with the aim to test the architecture. The collected real-time data passed to the server through an android application which evaluates the result based on the fuzzy rules set. Figure. 4(a) shows the health status to be critical when $\mathrm{SpO}_{2}$ value is $88 \%$ and heart rate is $55 \mathrm{bpm}$ (beats per minute), which is very low as per fuzzy calculation. Figure 4(b) shows the health status to be normal when $\mathrm{SpO}_{2}$ value is $98 \%$ and heart rate is $90 \mathrm{bpm}$ as per fuzzy calculation. Figure 4(c) shows how alert is displayed for a health

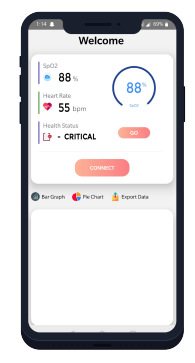

(A)

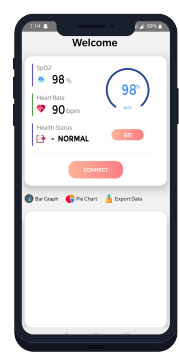

(B)

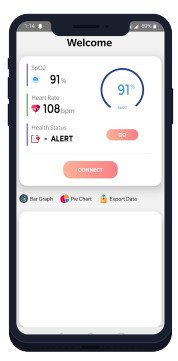

(C)
Figure 4: Real-time human health monitoring and processing result a) Critical b) Normal and c) Alert.

status when $\mathrm{SpO}_{2}$ value is $91 \%$ and heart rate is $108 \mathrm{bpm}$, which is obtained as per fuzzy calculation.

A dataset from Kaggle.com, comprising parameters such as heart rate, temperature and oxygen was taken into account. The dataset generated the result in boolean values where 0 indicated health to be critical and 1 indicated health to be normal.

In our study, two parameters were considered, namely $\mathrm{SpO}_{2}$ and heart rate, to determine health status. The dataset generated the result in three states, i.e. critical, alert and normal. In addition, the values of illness dataset were used in our study to acquire a realtime health status. The use of the illness dataset's values exhibited similar results to a great extent.

\section{CONCLUSION AND FUTURE WORK}

This study offered a cloud-based real-time application that monitored oxygen saturation and heart rate using fuzzy-as-a-service. A novel fuzzy logic algorithm was used to detect a human's medical condition with the help of a real-time wearable sensor and cloud-based web services. The sensor tracks a human's day-to-day activities or tasks. $\mathrm{SpO}_{2}$ and pulse rate were the two major factors that were considered from wearable sensors. The novelty that was followed in this study was that an IEEE1855-2016 algorithm was used in real-time along with fuzzy logic for managing sensor information. Moreover, the study included the use of fuzzy-as-aservice [15], [16] and real-time monitoring of activities, which were previously not conducted in wearable sensors.

As future work directions, the developed system can be extended to detect the symptoms of COVID-19 by utilising temperature, heart rate, and $\mathrm{SpO}_{2}$ sensors. Also, fuzzy services such as fuzzy querying about fuzzy databases or fuzzy ontologies can be considered for monitoring oxygen saturation and heart rate. Moreover, expanding web services to semantic web services would be beneficial due to the close association between fuzzy markup language and fuzzy ontologies.

\section{REFERENCES}

[1] Giovanni Acampora, Bruno Di Stefano, and Autilia Vitiello. 2016. IEEE $1855^{\mathrm{TM}}$. The First IEEE Standard Sponsored by IEEE Computational Intelligence Society [Society Briefs]. IEEE Computational Intelligence Magazine 11, 4 (2016), 4-6.

[2] Giovanni Acampora, Vincenzo Loia, and Autilia Vitiello. 2013. Distributing fuzzy reasoning through fuzzy markup language: An application to ambient intelligence. In On the Power of Fuzzy Markup Language. Springer, 33-50. 
[3] RR Adiputra, S Hadiyoso, and Y Sun Hariyani. 2018. Internet of things: Low cost and wearable $\mathrm{SpO} 2$ device for health monitoring. International fournal of Electrical and Computer Engineering 8, 2 (2018), 939.

[4] Mian Mujtaba Ali, Shyqyri Haxha, Munna M Alam, Chike Nwibor, and Mohamed Sakel. 2019. Design of Internet of Things (IoT) and Android Based Low Cost Health Monitoring Embedded System Wearable Sensor for Measuring SpO 2 , Heart Rate and Body Temperature Simultaneously. Wireless Personal Communications (2019), 1-15.

[5] Gözde Ateş and Kemal Polat. 2012. Measuring of oxygen saturation using pulse oximeter based on fuzzy logic. In 2012 IEEE International Symposium on Medical Measurements and Applications Proceedings. IEEE, 1-6.

[6] Frans M Coetzee and Ziad Elghazzawi. 2000. Noise-resistant pulse oximetry using a synthetic reference signal. IEEE Transactions on Biomedical Engineering 47, 8 (2000), 1018-1026.

[7] Yu Fu and Jian Liu. 2015. System design for wearable blood oxygen saturation and pulse measurement device. Procedia manufacturing 3 (2015), 1187-1194.

[8] Marek Krehel, Martin Wolf, Luciano F Boesel, René M Rossi, Gian-Luca Bona and Lukas J Scherer. 2014. Development of a luminous textile for reflective pulse oximetry measurements. Biomedical optics express 5, 8 (2014), 2537-2547.

[9] Ahmad Lotfi, Hamid Bouchachia, Alexander Gegov, Caroline Langensiepen, and Martin McGinnity. 2018. Advances in Computational Intelligence Systems. Intelligence (2018).

[10] Prisma Megantoro and others. 2020. Detection of Hypoxic Symptoms System Based on Oxygen Saturation and Heart Rate Using Arduino Based Fuzzy Method. In 2020 2nd International Conference on Industrial Electrical and Electronics (ICIEE) IEEE, 107-111.

[11] Abdallah Naser, Ahmad Lotfi, and Junpei Zhong. 2020. Adaptive Thermal Sensor Array Placement for Human Segmentation and Occupancy Estimation. IEEE Sensors fournal 21, 2 (2020), 1993-2002.

[12] Meir Nitzan and Haim Taitelbaum. 2008. The measurement of oxygen saturation in arterial and venous blood. IEEE instrumentation \& measurement magazine 11, 3 (2008), 9-15.

[13] World Health Organization and others. 2012. World health day: Are you ready? What you need to know about ageing. accessed December 20 (2012), 2012.
[14] Bhavesh Pandya, Amir Pourabdollah, and Ahmad Lotfi. 2020. Comparative Analysis of Real-Time Fall Detection Using Fuzzy Logic Web Services and Machine Learning. Technologies 8, 4 (2020), 74.

[15] Bhavesh Pandya, Amir Pourabdollah, and Ahmad Lotfi. 2020. Fuzzy-as-a-Service for Real-Time Human Activity Recognition Using IEEE 1855-2016 Standard. In 2020 IEEE International Conference on Fuzzy Systems (FUZZ-IEEE). IEEE, 1-8.

[16] Bhavesh Pandya, Amir Pourabdollah, and Ahmad Lotfi. 2020. Fuzzy logic web services for real-time fall detection using wearable accelerometer and gyroscope sensors. In Proceedings of the 13th ACM International Conference on PErvasive Technologies Related to Assistive Environments. 1-7.

[17] Mike Patton. 2015. US health care costs rise faster than inflation. Retrieved from Forbes: https://scholar. google. com/scholar (2015).

[18] Sita Radhakrishnan, Suresh G Nair, and Johney Isaac. 2019. Analysis of parameter affecting blood oxygen saturation and modeling of fuzzy logic system for inspired oxygen prediction. Computer methods and programs in biomedicine 176 (2019), 43-49.

[19] Muhammad F Rian, Michrandi N Surya, and Astuti N Ratna. 2019. Health monitoring application using fuzzy logic based on android. In fournal of Physics: Conference Series, Vol. 1192. IOP Publishing, 012052

[20] Ramya Shekar, N Sriraam, Prabhu Ravikala Vittal, and Uma Arun. 2020. A Wearable Wrist-Based Pulse Oximetry for Monitoring Cardiac Activities-A Pilot Study. In International Conference on Communication, Computing and Electronics Systems. Springer, 325-333.

[21] José M Soto-Hidalgo, Jose M Alonso, Giovanni Acampora, and Jesús Alcalá-Fdez. 2018. JFML: a java library to design fuzzy logic systems according to the IEEE std 1855-2016. IEEE Access 6 (2018), 54952-54964.

[22] Hariharan Subramanian, Bennett L Ibey, Weijian Xu, Mark A Wilson, M Nance Ericson, and Gerard L Coté. 2005. Real-time separation of perfusion and oxygenation signals for an implantable sensor using adaptive filtering. IEEE Transactions on Biomedical Engineering 52, 12 (2005), 2016-2023.

[23] Yuyang Xie, Yongjie Gao, Yuchun Li, Yu Lu, and Weixi Li. 2017. Development of wearable pulse oximeter based on internet of things and signal processing techniques. In 2017 European Modelling Symposium (EMS). IEEE, 249-254. 